\title{
F-18 fluorodeoxyglucose positron emission tomography for differential diagnosis and prognosis prediction of vascular tumors
}

\author{
WON WOO LEE ${ }^{1,2}$, YOUNG SO $^{3}$, SEO YOUNG KANG $^{1}$, MIN-KYUNG SO $^{1}$, \\ HAERYOUNG KIM ${ }^{4}$, HYUN WOO CHUNG ${ }^{3}$, WAN SEOP KIM ${ }^{5}$ and SANG EUN KIM ${ }^{1}$
}

\author{
${ }^{1}$ Department of Nuclear Medicine, Seoul National University Bundang Hospital, Seoul National University \\ College of Medicine, Seongnam, Gyeonggi-do 13620; ${ }^{2}$ Institute of Radiation Medicine, Medical Research Center, \\ Seoul National University, Seoul 03080; ${ }^{3}$ Department of Nuclear Medicine, Konkuk University Medical Center, \\ Konkuk University School of Medicine, Seoul 05030; ${ }^{4}$ Department of Pathology, Seoul National University \\ Bundang Hospital, Seoul National University College of Medicine, Seongnam, Gyeonggi-do 13620; \\ ${ }^{5}$ Department of Pathology, Konkuk University Medical Center, Konkuk University \\ School of Medicine, Seoul 05030, Republic of Korea
}

Received May 30, 2016; Accepted March 10, 2017

DOI: $10.3892 / \mathrm{ol} .2017 .6192$

\begin{abstract}
The spectrum of vascular tumors ranges from hemangioma (HEM), to epithelioid hemangioendothelioma (EHE) and to angiosarcoma (AS). To the best of our knowledge, the usefulness of F-18 fluorodeoxyglucose positron emission tomography (FDG-PET) for vascular tumors has never been comprehensively studied. The present study investigated the usefulness of FDG-PET for pathologically diagnosed vascular tumors. The present study included 26 patients with

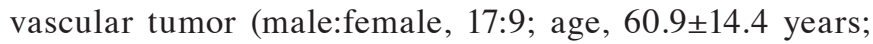
7 HEM, 6 EHE and 13 AS) who underwent FDG-PET between January 2007 and May 2014 at the Seoul National University Bundang Hospital (Seongnam, Korea) and Konkuk University Medical Center, (Seoul, Korea). Representative FDG uptake was measured as the maximum standardized uptake value (SUVmax) over the lesion with the highest FDG uptake. Disease progression was clinically defined as the aggravation of known lesions or novel lesion development during follow-up on computed tomography, magnetic resonance imaging, or FDG-PET. FDG-PET revealed multi-organ involvement only in AS (6/13 [46.2\%]), whereas HEM and EHE involved a single organ. Tumor SUVmax was significantly greater in AS $(6.32 \pm 4.84)$ compared with EHE $(3.10 \pm 2.68)$ and HEM $(2.33 \pm 0.76)(\mathrm{P}=0.0284)$. There was no difference in tumor
\end{abstract}

Correspondence to: Professor Young So, Department of Nuclear Medicine, Konkuk University Medical Center, Konkuk University School of Medicine, 120-1 Neungdong-ro, Gwangjin-gu, Seoul 05030, Republic of Korea

E-mail: youngso@kuh.ac.kr

Key words: angiosarcoma, epithelioid hemangioendothelioma, F-18 fluorodeoxyglucose positron emission tomography, hemangioma, vascular tumor
SUVmax between HEM and EHE $(\mathrm{P}>0.05)$. Disease progression was primarily noticed in AS (9/13 [69.2\%]). Only 1 patient with EHE $(1 / 6=16.7 \%)$ and no patients with HEM (0/7=0\%) experienced disease progression. Mortality was reported only in patients with AS (4/13 [30.8\%]). Using the cutoff SUVmax of 3.0, the two-year progression-free survival rate of 14 patients with tumor SUVmax $<3.0$ (75.0\%) was significantly higher compared with that of 12 patients with tumor SUVmax $\geq 3.0$ $(0 \%)(\mathrm{P}=0.0053)$. In conclusion, FDG-PET is useful for the differential diagnosis and prognosis prediction of vascular tumors.

\section{Introduction}

Vasculartumors constitute a diversity of endothelium-originated tumors, ranging from a completely benign tumor such as hemangioma (HEM), to an intermediately malignant epithelioid hemangioendothelioma (EHE) and finally to a highly aggressive angiosarcoma (AS) $(1,2)$. HEM is a benign malformation of the endothelium that requires no treatment. EHE is not aggressively treated in asymptomatic cases but local resection, chemotherapy and occasionally organ transplantation are required in symptomatic cases. Conversely, the highly malignant AS is the target of aggressive multidisciplinary treatment (3).

F-18 fluorodeoxyglucose positron emission tomography (FDG-PET) has been utilized to detect AS in a variety of regions, such as the scalp (4), cardiac right atrium (5), liver (6), extremities (7) and uterus (8). AS lesions were FDG-avid (4-8); therefore, response to chemotherapy was able to be successfully monitored using FDG-PET (4,7). However, the usefulness of FDG-PET in the management of AS is not clear, as only case reports are available in the literature.

With regards to EHE, there are certain contradictory studies regarding the utility of FDG-PET. EHE has been visualized using FDG-PET in the liver (9), bone marrow (10) and lung (11). However, in a recent study by Cazzuffi et al (12), 
no FDG uptake was identified in diagnosed pulmonary EHE. Similar to AS, the paucity of available data makes it challenging to determine the clinical usefulness of FDG-PET for EHE.

HEM is a benign vascular malformation, and anatomical imaging studies, such as computed tomography/magnetic resonance imaging (CT/MRI), often provide sufficient information for diagnosis (13). Regarding FDG-PET, there have been certain case reports describing incidental lesions with the presence or absence of FDG uptake (14). Additionally, the degree of FDG uptake in HEM ranges from mild to moderate across the literature $(15,16)$.

In the present retrospective study, patients with the three types of vascular tumors (HEM, EHE and AS) who underwent FDG-PET and pathological confirmation were enrolled. The clinical behavior of each vascular tumor was investigated based on FDG-PET data. The usefulness of FDG-PET was sought for in terms of the differential diagnosis and prognosis prediction of the three vascular tumors.

\section{Materials and methods}

Patients. A total of 26 consecutive patients with vascular tumors (male: female, 17:9; age, 60.9 \pm 14.4 years) who had undergone FDG-PET and pathological confirmation in Seoul National University Bundang Hospital (SNUBH) (Seongnam, Korea) and Konkuk University Medical Center (KUMC) (Seoul, Korea) between January 2007 and May 2014 (Table I) were enrolled. Tumor type was HEM in 7 patients, EHE in 6 patients and AS in 13 patients; 23 patients were screened at SNUBH and 3 patients were screened at KUMC. FDG-PET was performed prior to treatment in all patients. At SNUBH, a 64-channel multi-detector CT-integrated PET/CT scanner (DVCT; GE Healthcare Bio-Sciences, Pittsburgh, PA, USA) was used for 21 patients subsequent to 2009 and a dedicated PET scanner (Allegro; Philips Medical System, Cleveland, OH, USA) was used for 2 patients [1 with EHE (patient \#11) and 1 with AS (patient \#22)] diagnosed prior to 2009. At KUMC, a 2-channel multi-detector CT-integrated PET/CT scanner (GEMINI; Philips Medical System) was used for 3 patients [1 with EHE (patient \#13) and 2 with AS (patients\#16 and \#25)].Pathological confirmation was performed in all patients. The review boards of the two institutions approved the study design and waived the need for informed consent from the patients (IRB numbers: B-1505/300-101 and KUH1280075 for SNUBH and KUMC, respectively).

The 7 patients with HEM were all diagnosed by wide excision or excisional biopsy. They underwent FDG-PET, as other imaging studies of CT or MRI had revealed suspicious malignant or metastatic lesions; 3 of the 7 patients exhibited confirmed malignancy (1 rectal cancer, 1 lung cancer and 1 esophageal cancer). FDG-PET/CT scans were performed $110.57 \pm 190.09$ days prior to the pathologic diagnosis. Subsequent to confirming HEM, no additional treatment was administered to the patients.

The 6 patients with EHE were all clinically suspected of malignancy prior to pathologic confirmation. Pathologic diagnosis was achieved following immunohistochemical staining with anti-CD31 (cat. no., 131M-96; dilution, 1:200; Cell Marque; Sigma-Aldrich Co.; Darmstadt; Germany) and anti-CD34 (cat. no., 134M-15; dilution, 1:200; Cell Marque;
Sigma-Aldrich Co.; Darmstadt; Germany) antibodies in 4 of the EHE patients (patients \#8, \#9, \#11 and \#13). Sections $(4 \mu \mathrm{m})$ were made from the formalin-fixed, paraffin-embedded blocks of the representative tumor tissue. Immunohistochemical staining was performed with the automated XT iVIEW DAB V.1 procedure on the BenchMark XT (Ventana Medical Systems, Inc., Tucson, AZ, USA) using the aforementioned anti-CD31 and anti-CD34 antibodies. Antigen retrieval was performed with CC1 EDTA buffer (Ventana Medical Systems, Inc.) for 48 min of CD31 and for 32 min of CD34. Sections were incubated with primary antibodies for $32 \mathrm{~min}$ at $37^{\circ} \mathrm{C}$ for $\mathrm{CD} 31$ and $12 \mathrm{~min}$ at $37^{\circ} \mathrm{C}$ for $\mathrm{CD} 34$. Staining was detected with iVIEW DAB detection kit (Ventana Medical Systems, Inc.). Internal positive controls of endothelial cells were used. Substitution of the primary antibodies with immunoglobulin $\mathrm{G}$ was used as a negative control. The pathologist examined the slides using the light microscope (BX53; Olympus Corporation, Tokyo, Japan) and built-in software. FDG-PET was performed $9.0 \pm 7.75$ days prior to the pathologic confirmation. There was 1 patient with hepatic EHE who underwent liver transplantation following the pathologic diagnosis (patient \#8). The 3 patients with EHE with multiple lung nodules (patients \#10,\#12 and \#13) were diagnosed by wedge resection via thoracoscopy or percutaneous needle biopsy. No chemotherapy was administered to any of the patients with EHE, with the exception of one patient with hepatic EHE (patient \#8), following liver transplantation. Only 1 patient with breast EHE (patient \#9) exhibited a recurrent lesion at the previous excision site 15.9 months following the initial excision.

All the 13 patients with AS were clinically suspected of malignant disease at the initial presentation to the clinicians. FDG-PET was performed $20.9 \pm 64.0$ days prior to the pathologic diagnosis. The patients were managed using local treatment (resection, external radiotherapy, or transarterial chemoembolization) and/or systemic chemotherapy (paclitaxel; $60-80 \mathrm{mg} / \mathrm{m}^{2}$ intravenously on days $1,8,15$ every 4 weeks for 6 cycles; nexavar; $400 \mathrm{mg}$ twice daily orally for 4 weeks for 2 cycles; pazopanib; $800 \mathrm{mg}$ once daily orally for 4 weeks for 2 cycles). However, 9 patients with AS had disease progression and 4 of them succumbed despite treatment. Disease progression was defined as the growth of known hepatic lesions on CT with newly-developed ascites or hemoperitoneum (patients \#15, \#17, \#20 and \#26), progression of local scalp lesion (patient \#19), aggravation of metastasis at the first lumbar vertebra (L1) compressing the spinal cord on MRI (patient \#23), an increase of multiple lesions of lung, bone and pancreas on CT (patient \#21), an increase of lung lesion on CT (patient \#16) or newly-developed distant metastases on FDG-PET (patient \#18).

FDG-PET. There were two PET/CT scanners and one dedicated PET scanner employed in the present study. A total of 2 patients used a dedicated PET scanner (Allegro; Philips) (17), 3 patients used a 2 channel-CT integrated PET/CT (GEMINI; Philips) (18) and the remaining 21 patients used a 64-channel CT-integrated PET/CT scanner (DVCT; GE Healthcare Life Sciences) (19). FDG uptake was quantitated using the standardized uptake value (SUV). The maximum SUV (SUVmax) of the tumor was used to represent the tumor's FDG uptake. 

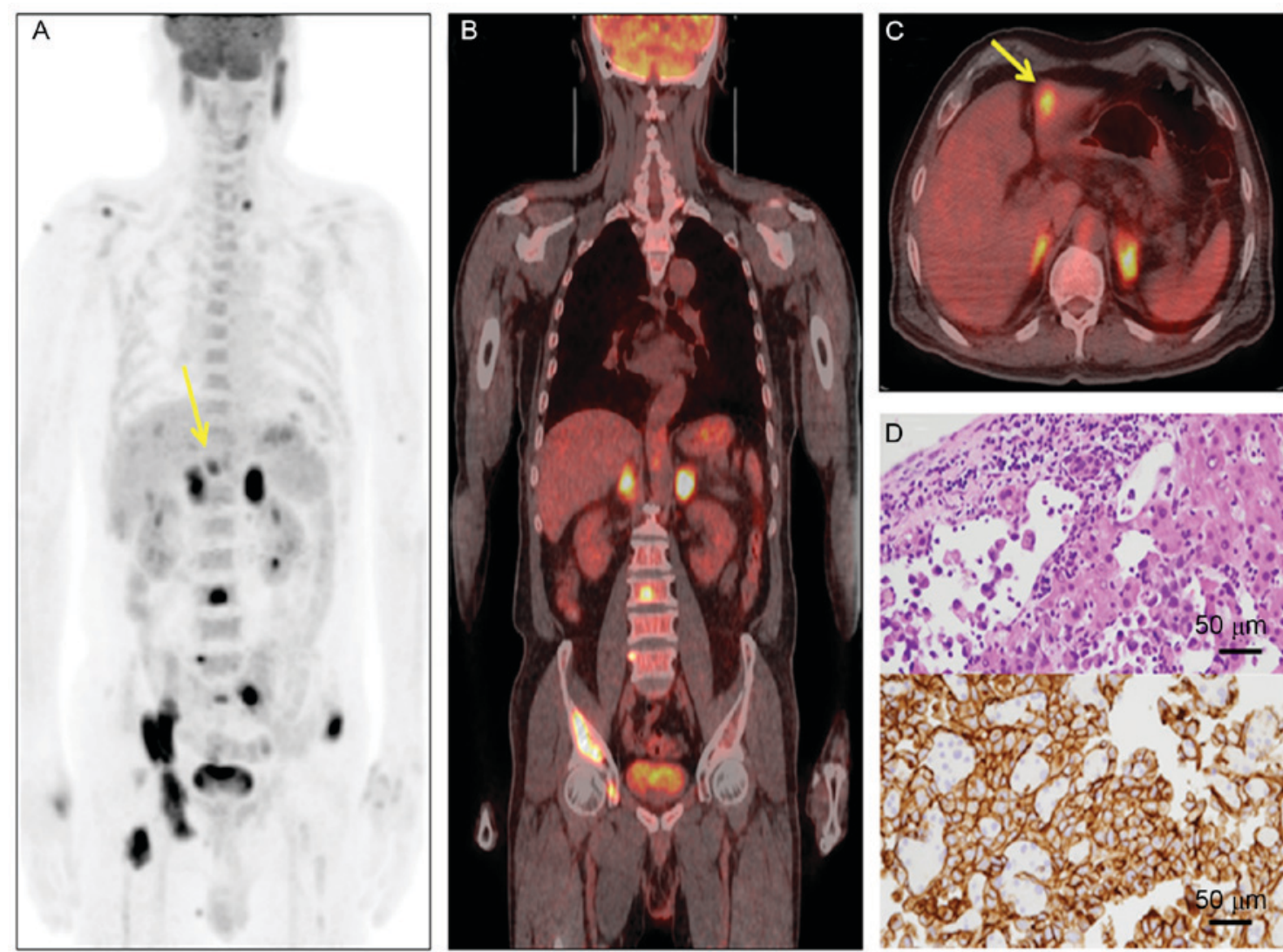

Figure 1. AS with multi-organ involvement on FDG-PET. A 68-year-old male patient (patient \#26) exhibited multiple hypermetabolic lesions in the liver, bilateral adrenal glands, stomach and multiple bones. Liver biopsy confirmed AS (yellow arrows). The highest maximum standardized uptake value was measured in the left adrenal gland as 11.6. (A) Maximum intensity projection anterior image. (B) PET/CT fusion coronal image. (C) PET/CT fusion transaxial image. (D) Pathology from liver biopsy demonstrating AS (magnification, x400). The upper panel demonstrates hematoxylin and eosin staining, and the lower demonstrates cluster of differentiation 31 staining. AS, angiosarcoma; FDG-PET, F-18 fluorodeoxyglucose positron emission tomography.

Image interpretation. FDG-PET images were interpreted by the attending nuclear medicine physicians. When consensus between the two readers at each institute was reached, the results were written down and analyzed. The readers observed the FDG-PET images with reference to all available clinical and radiological information. The most prominent lesion with the highest SUVmax was considered the representative lesion for each patient (Table I).

Clinical follow-up and disease progression. All the patients participated in follow-up imaging studies on a regular basis; every 3 months or at any time when disease progression was suspected. CT, MRI, or PET studies were requested according to the discretion of the physician. Disease progression was defined as aggravation of known lesions or novel lesion development during follow-up on CT, MRI or FDG-PET images. The time to progression was measured from the date of pathologic confirmation to the date of disease progression.

Statistical analysis. Statistical software (MedCalc version 12.7.1.0; MedCalc Software BVBA, Ostend, Belgium) was used for statistical analysis. A Kruskal-Wallis test was performed to compare the degree of FDG uptake among the vascular tumors. The difference of progression-free survival was investigated using a log rank test and Kaplan-Meier survival analysis. All data are presented as the mean \pm standard deviation. $\mathrm{P}<0.05$ was considered to indicate a statistically significant difference.

\section{Results}

Visual assessment of FDG-PET. Multi-organ involvement on FDG-PET was only observed in patients with AS (Fig. 1). In total, $6(46.2 \%)$ of the 13 patients with AS exhibited the involvement of more than one organ, whereas none of the patients with EHE or HEM demonstrated multi-organ involvement patterns on FDG-PET (Table I). A total of 3 patients with EHE exhibited multiple nodules in bilateral or ipsilateral lung parenchyma as a single organ involvement. FDG-PET illustrated mildly abnormal lesions in 2 patients with pulmonary EHE, but multiple hypermetabolic masses/nodules in 1 patient with pulmonary EHE (Fig. 2). The remaining 3 patients with EHE exhibited one single lesion on FDG-PET. A single lesion in a single organ was involved in patients with HEM (Fig. 3). The degree of FDG uptake on patients with HEM lesions was typically mild to moderate, and malignancy was not suspected in any patient with HEM by visual assessment alone.

Quantitative analyses of FDG-PET. Tumor FDG uptake, reflected by SUVmax, was compared among patients with HEM, EHE and AS. In patients who exhibited multiple FDG-avid lesions, the lesion with the highest SUVmax was considered the representative lesion. The SUVmax was significantly greater in patients with AS $(6.32 \pm 4.84)$ compared with EHE (3.10 \pm 2.68$)$ and HEM (2.33 $\pm 0.76 ; \mathrm{P}=0.0284)$. No 


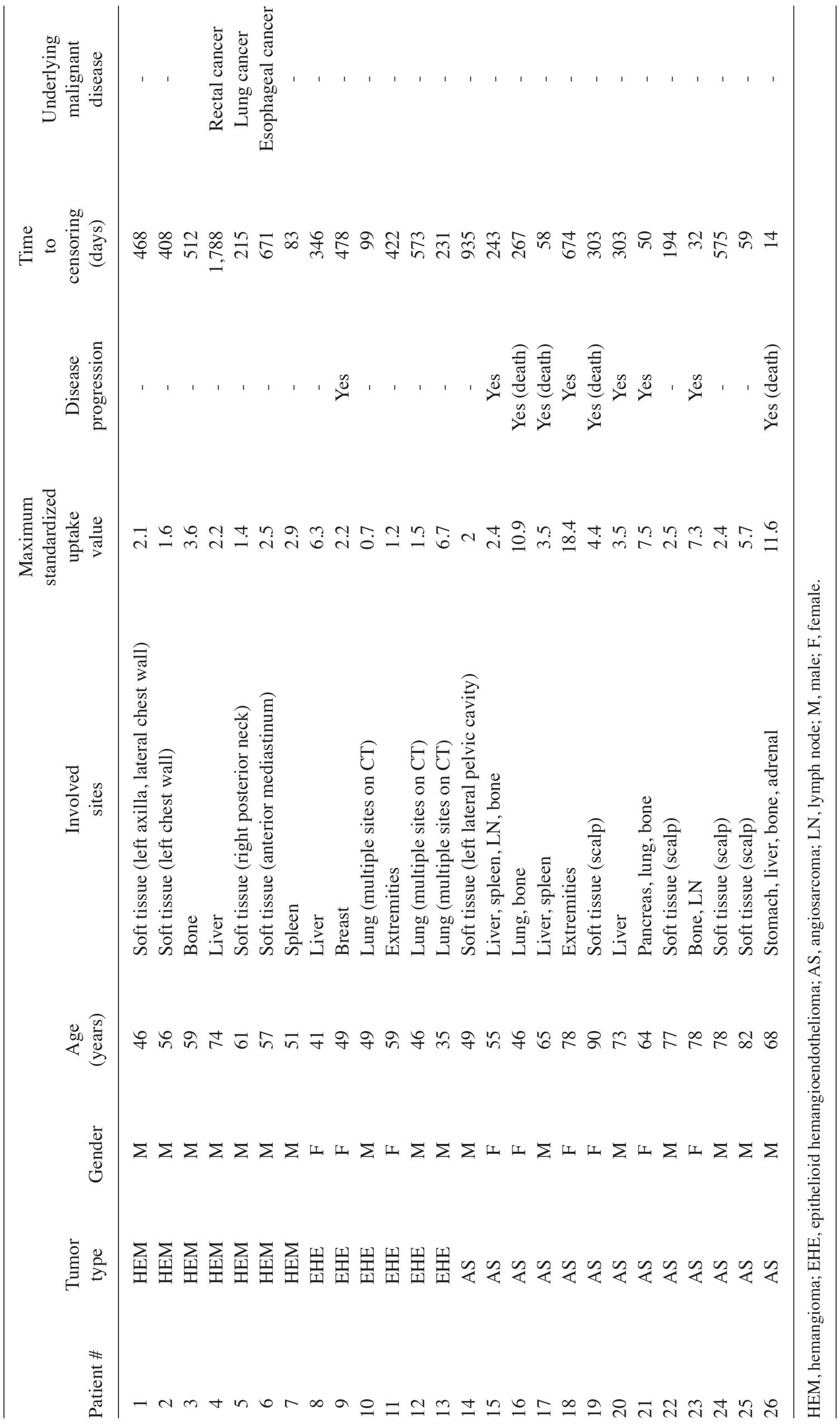



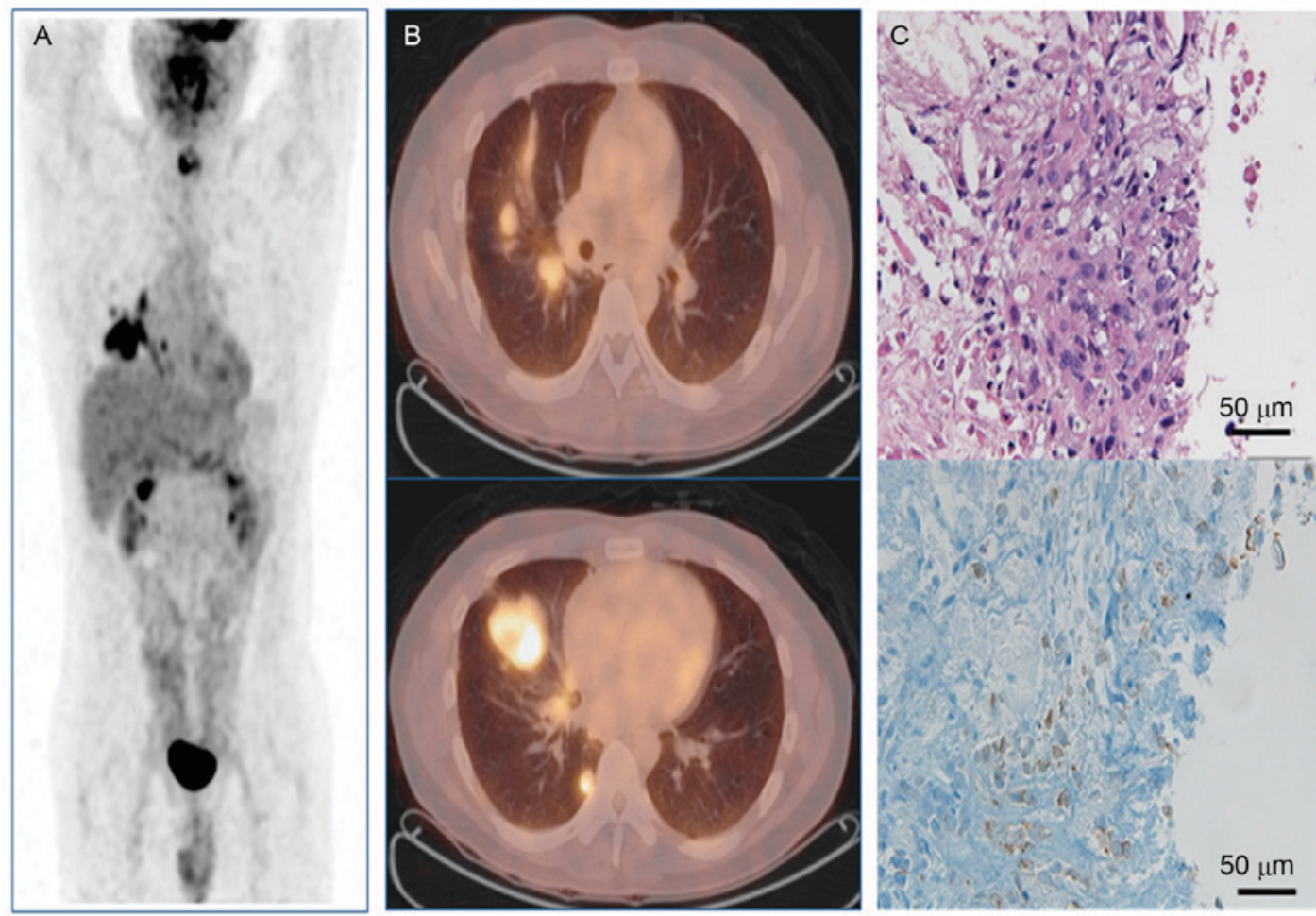

Figure 2. EHE with multiple lesions in the right lung on FDG-PET. A 35-year-old male patient (patient \#13) exhibited multiple hypermetabolic nodules in the right lung. Percutaneous needle biopsy over the right lung lesion revealed the EHE lesion. The highest maximum standardized uptake value of the right middle lobe lesion was 6.7. (A) Maximum intensity projection anterior image. (B) PET/CT fusion axial images. (C) Pathology from the percutaneous needle biopsy revealing EHE (magnification, x400). The upper panel demonstrates hematoxylin and eosin staining, and the lower demonstrates cluster of differentiation 31 staining. EHE, epithelioid hemangioendothelioma; FDG-PET, F-18 fluorodeoxyglucose positron emission tomography.
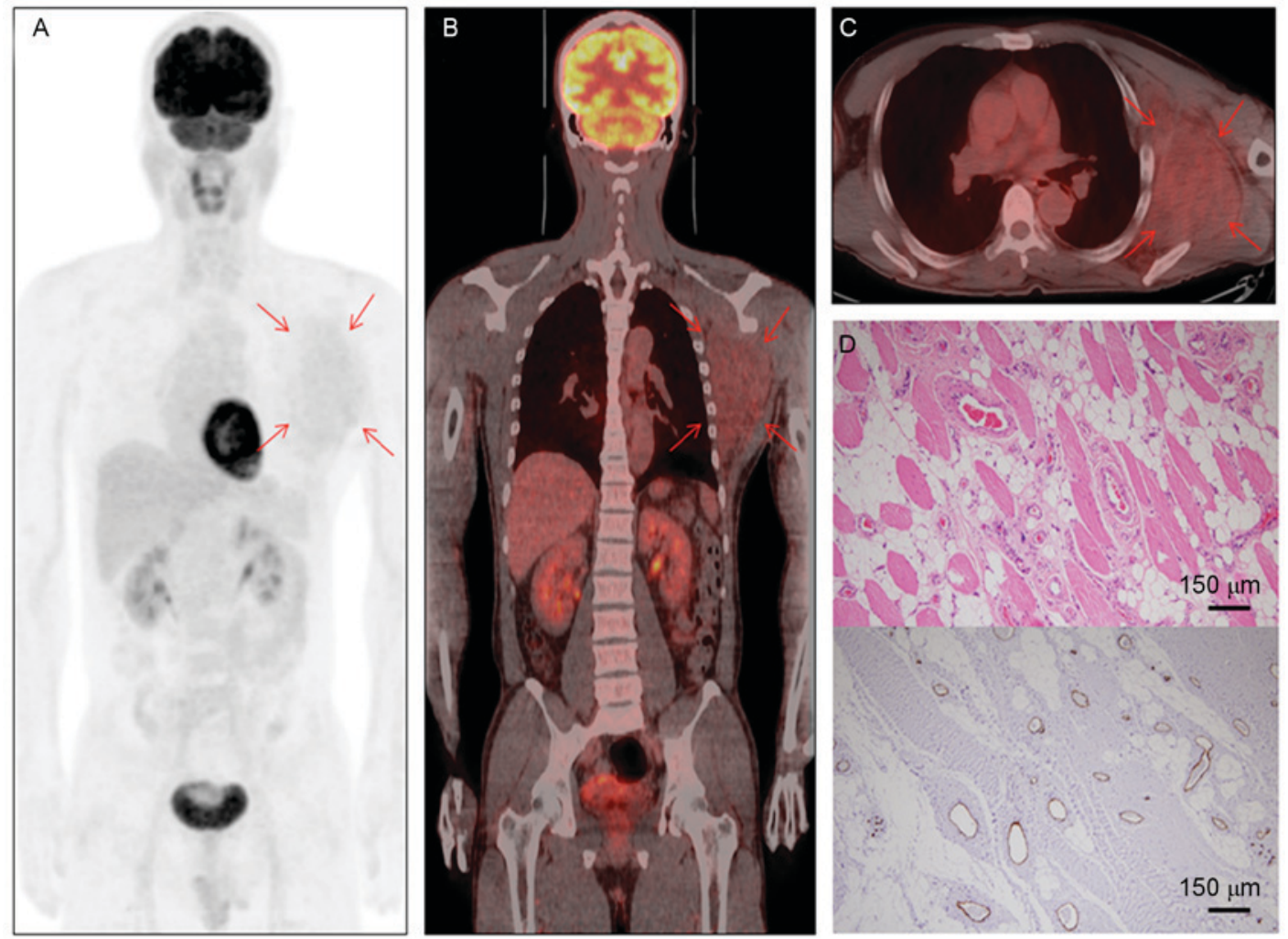

Figure 3. HEM with a single lesion with mild FDG uptake on FDG-PET. A 46-year-old male patient (patient \#1) exhibited a soft tissue mass in the left axilla that extended to the chest wall (red arrows). Maximum standardized uptake value of the mass lesion was 2.1. Wide excision confirmed the presence of HEM. (A) Maximum intensity projection anterior image. (B) PET/CT fusion coronal image. (C) PET/CT fusion transaxial image. (D) Pathology from left axilla demonstrating HEM (magnification, x100). The upper panel demonstrates hematoxylin and eosin staining, and the lower demonstrates cluster of differentiation 31 staining. HEM, hemangioma; FDG-PET, F-18 fluorodeoxyglucose positron emission tomography. 


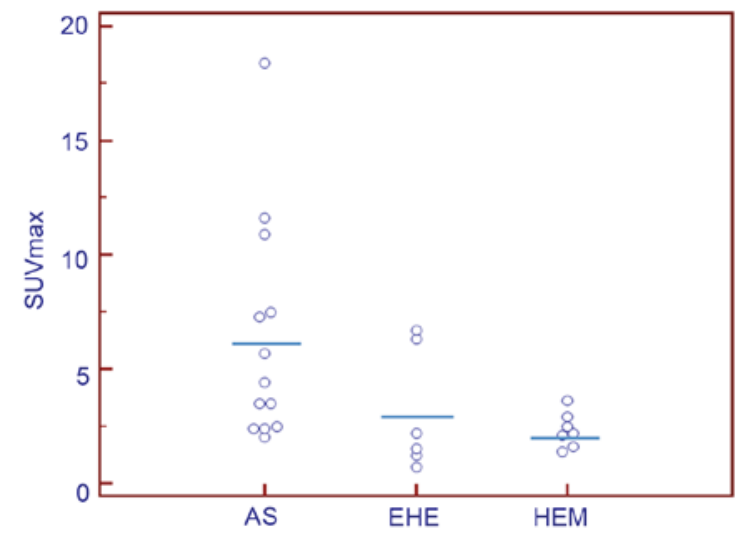

Figure 4. FDG uptake of the vascular tumors. AS exhibited a higher SUVmax (6.32 \pm 4.84$)$ compared with EHE (3.10 \pm 2.68$)$ and $\operatorname{HEM}(2.33 \pm 0.76 ; \mathrm{P}=0.0284$ by Kruskal-Wallis test). There was no difference between EHE and HEM $(\mathrm{P}>0.05)$. Horizontal bars indicate the mean value of SUVmax. AS, angiosarcoma; EHE, epithelioid hemangioendothelioma; HEM, hemangioma; FDG, fluorodeoxyglucose; SUVmax, maximum standardized uptake value.

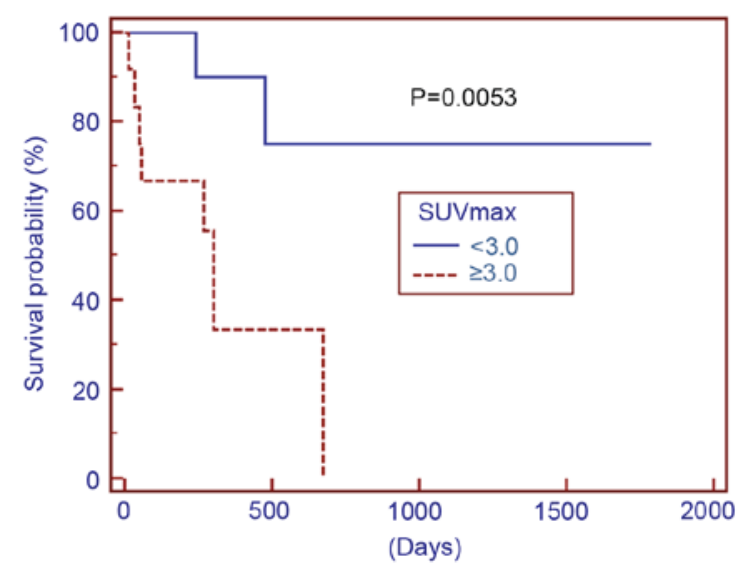

Figure 5. Survival curve analysis according to the cutoff SUVmax of 3.0 The 12 patients with higher SUVmax $(7.45 \pm 4.36)$ exhibited significantly poorer disease progression-free survival compared with the 14 patients with lower SUVmax $(1.97 \pm 0.61 ; \mathrm{P}=0.0053$ by log rank test). SUVmax, maximum standardized uptake value.

difference in tumor SUVmax was observed between patients with EHE and HEM (P>0.05; Fig. 4).

Prognostic prediction of vascular tumors by FDG-PET. Disease progression was observed in 9/13 (69.2\%) patients with AS, whereas only 1/6 (16.7\%) patients with EHE and 0/7 (0\%) patients with HEM experienced disease progression. Patient mortalities were only reported in patients with AS [4/13 (30.8\%)]. The SUVmax of 3.0 exhibited the most significant discriminating value for disease progression in receiver operating characteristic (ROC) curve analysis. Therefore, the patients with vascular tumors were dichotomized into two groups using the cutoff SUVmax of 3.0. One group comprised 12 patients ( 9 with AS and 3 with EHE; mean SUVmax $7.45 \pm 4.36$ ) with $S U V \max \geq 3.0$ and the other group comprised 14 patients (4 with AS, 4 with EHE and 6 with HEM; mean SUVmax 1.97 \pm 0.61 ) with SUVmax <3.0. Of the 12 patients with higher SUVmax, 8 (66.7\%) patients with AS experienced disease progression, including 4 mortalities, whereas 4 (1 with
AS, 2 with EHE and 1 with HEM) patients did not experience disease progression. Conversely, of the 14 patients with lower SUVmax, 2 (14.3\%) patients [1 with EHE and 1 with AS (no mortalities included)] had disease progression. The survival analysis demonstrated a significant difference between the two groups $(\mathrm{P}=0.0053$ by log rank test of Kaplan-Meier analysis; Fig. 5). The two-year progression-free survival rate of 14 patients with tumor SUVmax $<3.0$ was $75.0 \%$, whereas that of 12 patients with tumor SUVmax $\geq 3.0$ was $0 \%$.

\section{Discussion}

The spectrum of vascular tumors ranges from HEM to vascular sarcomas such as EHE and AS (1,2). Typical benign HEM and typical highly aggressive AS are the extremes of this vascular tumor spectrum. EHE exhibits intermediate characteristics, occasionally requiring treatments to ameliorate the disease progression, or occasionally being closely checked up for the serial changes without any treatment (3). In the present retrospective study, it was demonstrated that F-18 FDG-PET is useful for the differential diagnosis and prognosis prediction of different types of vascular tumors.

FDG-PET has been suggested to be useful for the diagnosis of vascular tumors in a number of case reports (4-11). However, a comprehensive analysis of the differential diagnosis, disease extent evaluation and prognosis prediction has never been performed. Due to the low incidence of AS, potentially $<2 \%$ of all soft tissue sarcomas $(1,2),>2$ patients with AS have never been evaluated by FDG-PET in a single study (7). Additionally, no previous FDG-PET study has compared AS with other vascular tumors such as EHE. The major results of the present study were as follows: i) AS involved multiple FDG-avid lesions/organs more frequently compared with EHE and HEM; ii) FDG uptake in AS lesions was greater compared with EHE and HEM and iii) the degree of FDG uptake, as reflected by SUVmax, was able to predict the prognosis of the patients with vascular tumors.

Regarding EHE, there are no consistent FDG-PET data in the literature: certain studies demonstrated high FDG uptake of EHE (9-11), but others did not (12). In the present study, of the 6 patients with EHE, 1 patient with hepatic EHE (patient \#8) and the other pulmonary patient with EHE (patient \#13; Fig. 2) demonstrated high FDG uptake (SUVmax, 6.3 and 6.7 , respectively) but disease progression did not take place following hepatic transplantation (patient \#8) or even in the absence of any treatment (patient \#13). There were 2 patients with EHE with multiple pulmonary nodules barely observed on FDG-PET due to low FDG uptake (patients \#10 and \#12). No disease progression was observed in the 2 patients with pulmonary EHE, either. However, 1 patient with breast EHE with mild FDG uptake (SUVmax, 2.2) exhibited recurrence at the location of previous resection 15.9 months subsequent to the local resection (patient \#9). These data may indicate that the indeterminate malignancy of EHE is reflected on FDG-PET. Aggressive treatment such as hepatic transplantation may have been able to successfully treat the patient with EHE with high FDG uptake (patient \#8). However, a patient with pulmonary EHE (patient \#13) with high FDG uptake did not exhibit disease progression during the 7.7 months of follow-up. Additionally, non-aggressive treatment such as 
local resection did not prevent disease progression of breast EHE with low FDG uptake (SUVmax, 2.2; patient \#9). In this regard, the role of FDG-PET for the treatment guidance of EHE needs to be clarified through additional studies.

HEM is a benign vascular malformation and does not require aggressive treatment. The specific diagnosis is usually achieved by biopsy or surgery and radiological studies often provide sufficient information about HEM. However, it is not always straightforward for the physicians to identify HEM with only imaging studies. In the patients with HEM in the present study, MRI and/or CT often produced inconclusive results, and malignant rather than benign lesions were suspected prior to pathologic confirmation, which was the reason why FDG-PET was requested for these patients. In these patients, FDG-PET always demonstrated low FDG uptake (Fig. 3), suggesting benign rather than malignant lesions. Therefore, FDG-PET may perform a crucial role for the identification of benign vascular tumors, such as HEM.

In the present study, the SUVmax of HEM and EHE was relatively lower compared with that of AS, but there was no significant difference between the two types of vascular tumors observed. Therefore, the discrimination between HEM and EHE may not be easy by analyzing the degree of FDG uptake alone. In terms of vascular tumor evolution, EHE is more likely to metastasize compared with HEM, but may not be as malignant as to result in patient mortalities: this may be reflected by the similar degree of FDG uptake between EHE and HEM. However, it is of note that the pattern and the number of FDG-avid lesions of EHE (Fig. 2) were often more aggressive compared with those of HEM (Fig. 3). Of course, an additional large-scale study is warranted to elucidate the clinical implications of FDG uptake between EHE and HEM.

The present study has several limitations. Not all the patients with vascular tumors were studied using the same PET or PET/CT scanner. This may have affected the SUV measurements. However, it is attested that the SUV differences among the state-of-the-art PET or PET/CT scanners are not so great, and that the overall tendency of tumor FDG uptake may be relevantly evaluated and compared (20). Once the 2 patients exclusively analyzed using PET (1 patient with EHE and 1 with AS) were excluded, SUVmax remained higher in AS (6.18 \pm 4.88$)$ compared with EHE $(3.48 \pm 2.81)$ and HEM $(2.33 \pm 0.76 ; \mathrm{P}=0.0371)$, and the survival difference using the cutoff of SUVmax of 3.0 remained significant $(\mathrm{P}=0.0094)$. When only the 23 patients from SNUBH were analyzed, SUVmax remained higher in AS (5.39 \pm 4.98$)$ compared with EHE (2.38 \pm 2.26$)$ and HEM (2.33 $\pm 0.76 ; P=0.0259)$, and SUVmax cutoff 3.0 remained a significant discriminator of progression-free survival $(\mathrm{P}=0.0044)$. Even when the analyses were restricted to the most homogeneous 21 patients from SNUBH with a single PET/CT scanner, the results continued to demonstrate the same tendency: SUVmax was higher in AS (5.71 \pm 5.17$)$ compared with HEH (2.68 \pm 2.49$)$ and HEM $(2.33 \pm 0.76)(\mathrm{P}=0.0458)$, and the survival difference using the cutoff of SUVmax of 3.0 remained significant $(\mathrm{P}=0.0080)$. Therefore, the difference of PET scanners may have exerted minimal effect on the results. An additional limitation was the small number of enrolled patients. Additional investigations with a larger number of patients are warranted.
In conclusion, vascular tumors such as AS, EHE and HEM may be discriminated according to the differences of degree and pattern of FDG uptake. Disease progression of the vascular tumors may be predicted using the SUVmax on FDG-PET. However, additional studies are required to investigate whether FDG-PET may have a role in guiding the treatment selection for vascular tumors.

\section{Acknowledgements}

The present study was supported in part by a grant from the Korean Health Technology R\&D Project, Ministry of Health \& Welfare, Republic of Korea (grant no., A111627-1101-0000100) and by grants from the National Research Foundation (NRF), Ministry of Education, Science and Technology (MEST), Republic of Korea (grant nos., 2012M2A2A7035589 and 2014M2B2A9030104).

\section{References}

1. Young RJ, Brown NJ, Reed MW, Hughes D and Woll PJ: Angiosarcoma. Lancet Oncol 11: 983-991, 2010.

2. Ravi V and Patel S: Vascular sarcomas. Curr Oncol Rep 15: 347-355, 2013.

3. Agulnik M, Yarber JL, Okuno SH, von Mehren M, Jovanovic BD, Brockstein BE, Evens AM and Benjamin RS: An open-label, multicenter, phase II study of bevacizumab for the treatment of angiosarcoma and epithelioid hemangioendotheliomas. Ann Oncol 24: 257-263, 2013.

4. Vasanawala MS, Wang Y, Quon A and Gambhir SS: F-18 fluorodeoxyglucose PET/CT as an imaging tool for staging and restaging cutaneous angiosarcoma of the scalp. Clin Nucl Med 31: 534-537, 2006.

5. Hori Y, Funabashi N, Miyauchi H, Nakagawa K, Shimura H, Miyazaki M, Kozono H, Nagai Y, Ishikura H, Nagai T, et al: Angiosarcoma in the right atria demonstrated by fusion images of multislice computed tomography and positron emission tomography using F-18 Fluoro-Deoxyglucose. Int J Cardiol 123: e15-e17, 2007.

6. Maeda T, Tateishi U, Hasegawa T, Ojima H, Arai Y and Sugimura K: Primary hepatic angiosarcoma on coregistered FDG PET and CT images. AJR Am J Roentgenol 188: 1615-1617, 2007.

7. Tokmak E, Ozkan E, Yağcı S and Kir KM: F18-FDG PET/CT Scanning in angiosarcoma: Report of two cases. Mol Imaging Radionucl Ther 20: 63-66, 2011.

8. Hwang JP and Lim SM: Uterine epithelioid angiosarcoma on F-18 FDG PET/CT. Nucl Med Mol Imaging 47: 134-137, 2013.

9. Nguyen BD: Epithelioid hemangioendothelioma of the liver with F-18 FDG PET imaging. Clin Nucl Med 29: 828-830, 2004.

10. Rest CC, Botton E, Robinet G, Conan-Charlet V, Bizais Y and Visvikis D: FDG PET in epithelioid hemangioendothelioma. Clin Nucl Med 29: 789-792, 2004.

11. Watanabe S, Yano F, Kita T, Soga S, Shinmoto H, Kosuda S, Ozeki Y, Aida S and Sakata I: 18F-FDG-PET/CT as an indicator for resection of pulmonary epithelioid hemangioendothelioma. Ann Nucl Med 22: 521-524, 2008.

12. Cazzuffi R, Calia N, Ravenna F, Pasquini C, Saturni S, Cavallesco GN, Quarantotto F, Rinaldi R, Cogo A, Caramori G and Papi A: Primary pulmonary epithelioid hemangioendothelioma: A rare cause of PET-negative pulmonary nodules. Case Rep Med 2011: 262674, 2011.

13. Sakurai K, Hara M, Ozawa Y, Nakagawa M and Shibamoto Y: Thoracic hemangiomas: Imaging via CT, MR, and PET along with pathologic correlation. J Thoracic Imaging 23: 114-120, 2008.

14. Choi YY, Kim JY and Yang SO: PET/CT in benign and malignant musculoskeletal tumors and tumor-like conditions. Semin Musculoskelet Radiol 18: 133-148, 2014.

15. Hatayama K, Watanabe H, Ahmed AR, Yanagawa T, Shinozaki T, Oriuchi N, Aoki J, Takeuchi K, Endo K and Takagishi K: Evaluation of hemangioma by positron emission tomography: Role in a multimodality approach. J Comput Assist Tomogr 27: 70-77, 2003. 
16. Ko SW and Park JG: Cavernous hemangioma of the ilium mimicking aggressive malignant bone tumor with increased activity on (18)F-FDG PET/CT. Korean J Radiol 14: 294-298, 2013.

17. Kang JY, Lee WW, So Y, Lee BC and Kim SE: Clinical usefulness of (18)F-fluoride bone PET. Nucl Med Mol Imaging 44: 55-61, 2010.

18. So Y, Yi JG, Song I, Lee WW, Chung HW, Park JH and Moon SG: Detection of skeletal muscle metastasis: Torso FDG PET-CT versus contrast-enhanced chest or abdomen CT. Acta Radiol 56: 860-866, 2015
19. Im HJ, Kim YK, Kim YI, Lee JJ, Lee WW and Kim SE: Usefulness of combined metabolic-volumetric indices of (18)F-FDG PET/CT for the early prediction of neoadjuvant chemotherapy outcomes in breast cancer. Nucl Med Mol Imaging 47: 36-43, 2013.

20. Fendler WP, Lehmann M, Todica A, Herrmann K, Knösel T, Angele MK, Dürr HR, Rauch J, Bartenstein P, Cyran CC, et al: PET response criteria in solid tumors predicts progression-free survival and time to local or distant progression after chemotherapy with regional hyperthermia for soft-tissue sarcoma. J Nucl Med 56: 530-537, 2015. 